Hirsch E and colleagues (Strasbourg, France) report a significant relationship between a slow EEG focus and neuropsychological impairment, in a prospective study of 18 children with benign epilepsy with centro-temporal spikes (BECTS). Low performance on Koh's cube and WISC coding test correlate with the amount of spike and wave on sleep EEGs. (Abstracts frtom the Annual Meeting of the AES, Los Angeles, CA, December 1-6, 2000. Epilepsia Oct 2000;41:Suppl 7; 88).

Caplan $\mathrm{R}$ et al (UCLA, CA) studied thought disorder in 92 children with complex partial seizures (CPS), 51 with petit mal (PGE), and in 117 normal children, ages 5 to 16 years. The CPS group had more severe thought disorder and cognitive impairments than the PGE group. EEG evidence of fronto-temporal dysfunction in children with CPS was associated with thought disorder as well as global cognitive dysfunction. In the PGE group, thought disorder was related to poor seizure control and cognitive dysfunction. (Abstracts from the Annual Meeting of the AES, Los Angeles, CA, Decemiber 1-6, 2000. Epilepsia Oci 2000;41:Suppl 7;88).

\title{
SELF-ESTEEM IN LEARNING-DISABLED CHILDREN WITH ADHD
}

The level of self-esteem was measured, using the Piers-Harris Self-Concept Scale, in 143 special education students at high risk for ADHD in the school year 1995, at a school district in Northern Florida. Overall, self-esteem scores were in the normal range. Children with ADHD and internalizing symptoms (anxiety or depressive disorders, diagnosed in 29\%, according to child self-report questionnaires) had significantly lower self-esteem scores, compared to children with ADHD alone or ADHD with comorbid disruptive behaviors. Those with higher levels of functional impairment were also at greater risk for low self-esteem. Children from minority backgrounds, primarily African-American, had higher self-esteem scores than white children. Medication use was not an independent predictor of low self-esteem. (Bussing R, Zima BT, Perwien AR. Self-esteem in special education children with ADHD: relationship to disorder characteristics and medication use. LAm Acad Child Adolesc Psychiatry October 2000;39:1260-1269). (Respond: Dr Regina Bussing, Box 100177 UFHC, Gainesville, FL 32610).

COMMENT. Comorbid internalizing (anxiety or depression) symptoms and severe learning or emotional dysfunction can predict low self-esteem in ADHD children. Minority background and use of stimulant medication do not increase risk of low self-esteem. In fact, African-American children with ADHD have a relatively higher level of self-esteem than their white counterparts.

\section{REGIONAL BRAIN VOLUME AND COGNITIVE OUTCOME IN PRETERM INFANTS}

Regional cortical volumes, measured by structural magnetic resonance imaging scans, were compared in 25 eight-year-old preterm children and 39 term control children, in a study performed at Yale and Brown University Medical Schools, and reported from the Yale Child Study Center, New Haven, CT. Regional cortical volumes were significantly smaller in the preterm children, especially in sensorimotor areas, but also in premotor, midtemporal, parieto-occipital, and subgenual cortices. Preterm children had significantly larger occipital and temporal, ventricular horns, and smaller volumes of cerebellum, basal ganglia, amygdala, hippocampus, and corpus callosum. The lower volumes of sensorimotor and midtemporal cortices in preterms were correlated with impaired full-scale, verbal, and performance IQ scores. (Peterson BS, Vohr B, Staib LH et al. Regional 